Unity Journal

Vol. III, 245-252, 2022

Doi: https://doi.org/10.3126/unityj.v3i01.43329

Prithvi Narayan Shah Research Center

Directorate General of Military Training, Nepali Army

Kathmandu, Nepal

\title{
Augmented Intelligence for National Security and Development
}

\section{Sristi Suman}

\begin{abstract}
Augmented Intelligence is a system model which centre on the partnership between human general intelligence \& common sense with Artificial Intelligence's exceptional processing speed and computation power to get better cognitive performance. This study can shed light on Augmented Intelligence and how it can be implemented so that we have heroes working at the peak human level possible, suffer fewer casualties, and also have equal footing in terms of technology and military strength. Nepal being open bordered country has a great threat from the border issues and with ever-rising crime and challenges in various cities creating intra threat. The study analyses to overcome this geographical challenge, operational problems, the rise of conflicts, and establish better policies, faced by security personnel with technology that actually empowers all the brave soldiers and Police officers to maintain security from both external and internal risk. In the ever-evolving technology limitations of human beings can be catastrophic, but replacing it all with Artificial Intelligence is not a practical solution as well because it would be giving the third entity all the ability that might revolt against humans themselves. The article can be summed up as, research on the practicality of Artificial Intelligence
\end{abstract}

and Augmented Intelligence in national security and figure out a way for its legal adaptation. The availability of various case studies, surveys, research on various existing technology, and research papers published in renowned journals and publications has made this study possible.

Keywords: Augmented Intelligence, exceptional processing speed, technology, military strength, Artificial Intelligence, defence, and security.

\section{Introduction}

Augmented Intelligence is a type of technology combining a common sense of humans with the computational power of Artificial Intelligence. This Technology has been preferred throughout the world for all different kinds of sections and the technology itself can be used for the defense of the nation and implementation of laws and policies for the nation. Augmented Intelligence is an enthralling technology. It will be the most powerful tool for benefiting humanity in generations. Its function and popularity are growing by the day. Machines are becoming increasingly adept at data analysis. But many jobs are inaccessible to machines. Augmented intelligence can be used in different fields like Medicine, business, border security, cybersecurity, education, and different 
organizations for the development and security of a nation. Smart algorithms that provide fast, data-driven predictive insights can supplement human instinct.

Artificial Intelligence has been the new frontier for technological breakthrough for the past decade with the evolution of smarter systems being more and more independent and capable of making big decisions but when we hit the pinnacle of artificial intelligence there will come a point where the Intelligent Agent becomes completely independent and start making drastic decisions that reward its own gains but can be catastrophic for the society hence many have believed that Artificial Intelligence will be the final step of human evolution. But creating a system that is dependent on human beings yet is capable of performing at utmost efficiency makes it a much more secure and safe technology than Artificial Intelligence. Hence instead of making machines more capable with complex algorithms Augmented Intelligence focuses on making humans more capable with the help of machines.

Stanford's Human-Centered Artificial Intelligence Institute, launched in March 2019, defines augmentation as practices that further the human condition, not diminish it (Bates, 2020). In April, Amazon Web Services announced its latest AI initiative called Augmented Artificial Intelligence (A2I), which promises to streamline humanAI interplay across a wide variety of fields (AWS, 2019). From 2021 to 2030, the global augmented intelligence market is expected to increase at a CAGR of 26.4 percent, from $\$ 11.73$ billion in 2020 to $\$ 121.57$ billion in 2030. North America dominated the augmented intelligence market in 2020 and is expected to maintain its position during the forecast period. In addition, the presence of an increasing number of cloud augmented intelligence solution vendors across the United States and Canada is expected to provide lucrative opportunities for the market. The augmented intelligence market is expected to thrive in the COVID-19 scenario, owing to various government, public, and other organizations adopting a work-fromhome culture for their employees.

For a developing country like Nepal is affected by all the innovations and new technology. Adopting Augmented intelligence and Artificial Intelligence is critical for Nepal where large data collection is still difficult. We may be able to make more accurate predictions about where the problems may actually lie if we use the array of data sources. Even though, this article discusses "what impact Augmented Intelligence can make for Nepal?" in terms of security and development. This is a difficult question to answer as it is determined by a variety of factors, the most important of which is the government's policy on information technology and related fields. And also describe Artificial Intelligence's difference with Augmented Intelligence, how does it work, existing systems, and their efficiency.

\section{Literature Review}

The concept of Augmented Intelligence and Artificial Intelligence is defined by a number of theories and findings for national security and development along with various projects representing the same concept in real-world scenarios. The different state of research includes Scopus, web science, and google scholar. The search yields more than 900 google scholars, more than 10 webs of science, and Scopus databases yielded more than 30 results related to AI and Augmented intelligence. Most of the publications came 
from the conference than journals. Some of them include publications from Nepal as well.

World Bank (2021) explores some of the approaches to AI across 11 countries at different stages of digital maturity and economic development, mapping initiatives to different policy domain areas and identifying the tools and instruments that have been proposed to implement AI strategies (Bank, 2021). Kokkalis, Konstantinos explains how this thesis additionally sets the base for further research in the fields of experimentation and legal adaptations to keep pace with this quite promising but also a quite challenging domain of AI. Furthermore, the thesis provides legal and technical recommendations for the potential implementation of an AI-embedded Command and Control system (Kokkalis \&Konstantinos, 2012). A novel approach towards augmenting artificial and human intelligence with the goal of enhancing the capabilities of human activity using adaptive intelligent agents and deep neural networks. Any intelligent system would have come across a situation where human intervention is essential; wherein human intelligence is required for the complete functioning of the agent (Hebbar, 2017). The paper AI 2.0: Augmented Intelligence written from a computer science and engineering perspective, focuses on the technical aspects of augmented intelligence; nevertheless, because of its broad scope, it is also a fascinating topic for humanities and social sciences to study (Lisboa, 2018). Augmented Intelligence - the new AI-unleashing human capabilities in knowledge work, here the authors examined augmented intelligence in trans humanistic categories, emphasizing the importance of this technology for human development. The other publication like what's trending in libraries from the Internet cybersphere - artificial intelligence and other emerging technologies through very interesting and useful in the general understanding of the role of AI in libraries, it does not reflect the specifics of augmented intelligence (Oyelude, 2017).

In the context of Nepal, Border management seeks to secure the state first and then maintain interstate relations (Upreti, 2021). Nepal's border has been plagued by crime, natural disasters, and bilateral disputes with its neighbors. According to the Center for Strategic and International Studies (2010), "trained border police," "sufficient modern equipment," and "compatible bureaucratic relationships" are required for smooth and professional border security. Nepal needs to start growing its own AI expertise to help develop national or South Asian solutions. This would require investing in local resources such as access to computer power or capacity as well as training young Nepali to work in AI (van Teijlingen A, 2020).

It can be concluded that the topic of Augmented Intelligence is generally scattered in most of the conference materials. Practical implementation of Augmented Intelligence is seen fewer than Artificial Intelligence. The countries like the USA, UK, Germany, and China are at the top in terms of Artificial intelligence but developing country like Nepal is still behind. So, this paper will discuss how it can be embraced and what will be the impact on the social, economic, political, and geographical parts of Nepal.

\section{Methodology}

The main purpose of this paper is to point out how AI and Augmented Intelligence can be adopted fornational security and development. 
So to meet the objectives of the study, the key approach were mostly qualitative. The study makes use of both primary and secondary sources of data. Secondary data are collected from different publications, journal articles, books, online search engines, documentaries, and newspapers. The primary data include observations, interviews with international experts, and customs officers. And systematic searches for specific questions were conducted using the scientific databases Scopus and Google Scholar, as well as Web of Science. Data from these various sources have been compiled to form a proper analysis and conclusion.

\section{How does Augmented Intelligence work?}

Augmented Intelligence gathers all type of data (structured and unstructured) from many sources, across disparate and siloed systems and they convert the data into reliable information and provide it to the users. For Instance, an Augmented Intelligence assisted Visor is capable of scanning the surroundings use the real-time feed to convert into multiple still frames (images-unstructured data) use complex algorithms and neural nets to classify all the objects in the line of sight and label them all to the user making it tactically safer for soldiers to navigate through battlefields and hostile territory. This is just one of many examples of Augmented Systems helping the users.

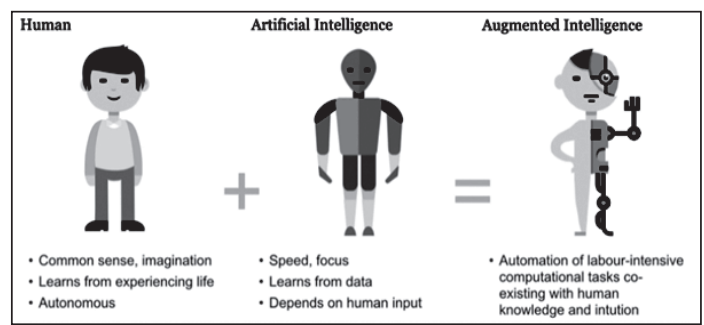

Source: https://medium.com/quantumblack/ operating-at-the-boundaries-augmentedintelligence-43e73684067d

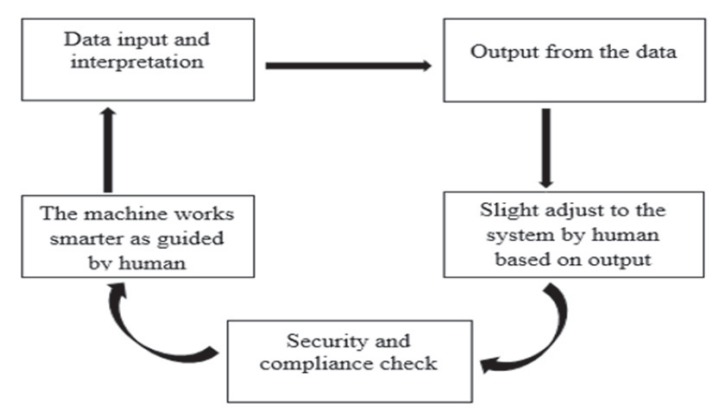

Fig 1: Diagram Representing Augmented Intelligence

\section{Current scenario of Nepal in Development and Security}

Nepal being a developing country, especially in the field of technology has not taken any strides towards implementation of augmented intelligence in development and security but that also means big strides and huge leaps can be taken and is bound to be taken at one point or the. For Better reflecting the scenario this article describes various different sectors, what is the current scenario and how it can be enhanced using Augmented Intelligence.

\section{Military Sector:}

The Military Sector is the one sector where Augmented Intelligence can be used to its peak potential enhancing all the soldiers to their utmost efficiency. In the current scenario Augmented intelligence has not made big strides in this sector. There has been some development like facial recognitionbased surveillance system that has been developed on various military projects along with securing encrypted files using facial recognition and other deep learning methods this has helped secure information in military sectors and also have a decent working surveillance system. There is huge room for improvements implementing various ideas of augmented intelligence as: 
- Smart weapons with Artificial Targeting mechanism and biometrics intelligence capable of locking the weapons when someone else then the owner of the weapon wields it.

- Smart Visor capable of recognizing various patterns and feeding the information to the soldier in fields, Visor capable of configuring the wiring of bombs and assisting in predicting which wire to be disconnected to diffuse it.

- Pattern analysis and decision-making expert system capable of predicting the best tactical plan to be implemented and give as much information as it can like an actual expert in the battle field which can be consulted by generals to make huge tactical decisions.

\section{Civil Sector:}

Artificial Intelligence has taken a great deal of improvements on the civil sector due to booming of AI Engineers and companies centered around Artificial Intelligence in Nepal like Fuse Machines, Augmented Intelligence however still a field that can be improved greatly is.

Artificial Intelligence itself has been used in the civil sector in various fields such as the smart restaurant where Robots would serve the guests visiting the hotel, Navigation of pathao service uses Artificial Intelligence to compute the shortest path and also calculate the fare for each trip. In terms of Augmented Intelligence, there have not been many developments.

Augmented Intelligence can be used for the civil sector of Nepal with the following possibilities:
- Stock Predictor and expense Estimator System can be used by various shops and customers to predict how much expense is going to take place and what products need to be stocked for a season this will be augmented Intelligence as it recommends the best action to the user instead of taking action itself.

- Smart Glasses are capable of navigating the path reading various languages and translating them as well. This will provide tourist's better guidance as these glasses will convert any given language to any other language as required.

\section{Impact of Augmented Intelligence in Development and Security}

It will be difficult to address the ethical and trust-related implications and risks associated with the integrity and security of these systems but it is necessary if the potential benefits of AI for defense are to be fully realized. If Nepalese defense organizations can strike the right balance, this new technology has enormous potential for mitigating some of the negative effects of conflict. It will improve intelligence gathering and analysis speed in command functions, logistic system automation and optimization, AI support for medical diagnosis and treatment; and a reduction in the number of human personnel deployed to dangerous environments and missions.

Opportunity spaces in Defence and Security: Military, Police and Border security

Augmented Intelligence allows us to Integrate Artificial Intelligence technology into collection interpretation and enhancing cognition between user and machine getting input from a lot of receptors like Drone 
Surveillance feed and satellite data which helps people make the most rewarding decisions that can be taken on the field. Using autonomous vehicles, such as submarines and land machines, enhanced with reinforcement learning algorithms to perform reconnaissance and discover targets with humans controlling all the decisive action gives a significant advantage compared to completely different entities of Artificial Intelligence. Virtual monitoring of borders using unmanned autonomous systems provides real-time monitoring of the activities across the borders. With security forces, the scale of technologies is unparalleled. Border data will be monitored in real-time and automatically fed into predictive analytics, machine learning, and artificial intelligence to generate actionable alerts so that security forces can intervene. Such alerts can be set off in response to the movement of physical objects as well as data on the internet. Robotics and autonomous systems can be used in a variety of civil domains, particularly as technological advancements make them less expensive and bulky while also making them more flexible and easier to interact with. Robots and swarm systems have the potential to play a disruptive role in military operations in the near future, as they will enable humans to perform tasks that are currently considered too risky, complex, or even impossible for humans to perform.

Artificial Intelligence technology combines environmental, asset, and historical mission data to better predict mission scenarios to ensure better data and information provided to users which can be used to make critical decisions. Such as having AI Technology to scan the terrain and recommend the best strategies that can be taken to military leaders give huge advantage to the entire platoon.
Enhanced Cognition with Deep learning technology incorporating sensor technology and computer vision to detect flaws and system failures in equipment before they occur. This can help avoid many fatal accidents from occurring and save many lives in battle field such as having visors capable of assessing the situation of weapons such that they can provide information using Augmented Reality in visor suggesting that the weapon won't work at all. Hence human soldiers will learn to rely more on intelligent systems that can think faster than they can, detecting threats before they do.

Implementing Artificial Intelligence technology with surveillance systems can be used to better anticipate threats and scenarios with Police captains being informed about all the surveillance reports to better deploy police resources in order to ensure community safety and supporting staff are utilized effectively. Using drone technology and imagerecognition capabilities to locate missing persons, manage crime scenes, and support forensic investigations. With a constant stream of all the results to reporting officers makes it easier for Police in fields to act on that information. The ability to scan images from millions of CCTV cameras provides a competitive advantage in surveillance and counterinsurgency operations; being able to track multiple potential targets; using big data to make increasingly accurate predictions of a target's behavior. So for better detection of suspicious activity, AI-powered systems will be best to analyze trends and patterns of behavior in traveler data. Another nation security technology may be the biometric passport. Using biometric technology and facial recognition software can provide numerous benefits, such as improving passenger flow in airports and re-directing 
security personnel to only address specific individuals. A biometric passport includes all of the biographical information found in a machine-readable document, as well as specific biometrics. The Entry-Exit System (EES), a key component of ETIAS, will use this biometric data to prevent irregular migration and protect Nepalese citizens. EES will use biometrics to identify over stayers and prevent identity fraud by registering the arrival and departure of third-country nationals.

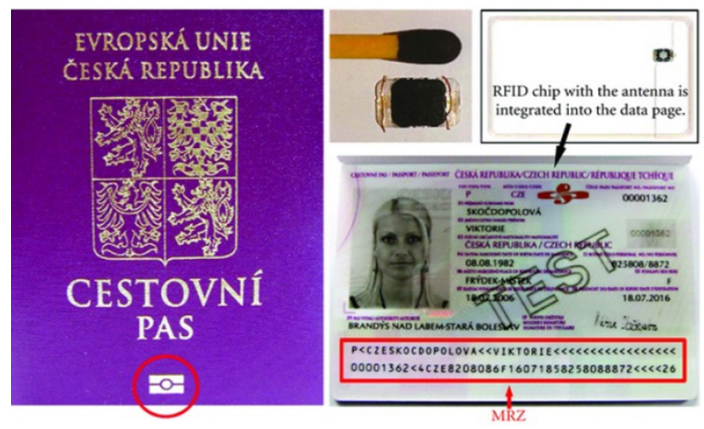

Fig 1.2: Biometric Passport

Source: https://www.researchgate.net/figure/ On-the-left-a-Czech-biometric-passportspecimen-with-circled-international-logoof_fig1_230833676

Conclusively, the future of Nepalese travel is inextricably linked to advances in artificial intelligence and Augmented Intelligence. It appears that, rather than jeopardizing security, digitalization will make Nepal safer than ever before for both residents and visitors.

\section{Result and Discussion}

\section{Challenges adopting Artificial Intelligence and Augmented Intelligence}

The biggest challenge for defense and security will be transitioning these technologies from the lab to real-world operations where robots must coexist and cooperate with humans where robots will have to co-exist and cooperate with humans. The successful implementation of autonomous systems in defense does not rely solely on technological advancement. Also, the military operates in a closed network, making access to information via new technologies more difficult. Political, cultural, sociological, and regulatory issues, as well as potential ethical and legal implications and safety constraints, must all be considered, particularly when autonomous systems are armed with lethal weapons.

The geography and topography of Nepal pose a physical security challenge but has the potential to serve as a testing ground for cutting-edge border technologies. Collecting large amounts of data in Nepal also entails thinking about the data's quality and where it will be stored so that it can be used. Because of these technical infrastructure constraints, AI implementation becomes difficult. Furthermore, although police departments are eager to work with AI, there is a natural tension between adopting this technology and ensuring the proper governance and framework are in place to avoid unintended consequences.

To address current border management issues, financing and developing next-generation unified border security management policies is critical. The Nepalese government should develop a unified national security policy that includes all law enforcement agencies in order to build a national intelligence information system. So organizations across government bodies, including the military, police services, and border security agencies, can institutionalize Artificial Intelligence and Augmented Intelligence solutions that are governed with the highest level of accountability, explicability, and success with right change in management strategy. 


\section{Conclusion}

Conclusively this study found out in detail about what Augmented Intelligence is, how it differs from Artificial Intelligence, how all over the world investment for Augmented Intelligence has been increasing, and how it can be implemented on various levels such as military operations, police operations, security and maintaining stability. Also found out how big players have been racing for investment in Artificial Intelligence to create the most balanced Augmented Intelligence with enhanced cognition using advanced Artificial Intelligence in the context of Nepal.

The future of technology appears to be Augmented Intelligence, not Artificial Intelligence for the development and security of the nation. Apart from military and border security, every other major sector involving large-scale human interactions stands to benefit from Augmented intelligence. Hence it has the potential to significantly benefit industries such as healthcare, education, banking, insurance, and government services. So Nepal must reframe its mind-set of the small country syndrome and bring new technology to the country's development

\section{References}

World Bank. (2021, May). Harnessing Artificial Intelligence for Development on the PostCOVID-19 Era : A Review of National AI Strategies and Policies. World Bank Group. from World Bank Group: http://hdl.handle. net/10986/35619

Bates, A. (2020, May). The State of Augmented Intelligence. Forbes. Retrieved from https:/www.forbes.com/sites/ forbestechcouncil/2020/05/21/the-state-ofaugmented-intelligence/?sh $=717 \mathrm{~d} 01204 \mathrm{e} 05$
Hebbar, A. (2017). Augmented intelligence: Enhancing human capabilities. Research Gate. doi:10.1109/ICRCICN.2017.8234515

Kokkalis, \& Konstantinos. (2012, December 19). Contribution of Artificial Intelligence to Border Security. Naval Postgraduate School. http://hdl.handle.net/10945/63998

Lisboa, P. (2018). AI 2.0: Augmented Intelligence. 13th International FLINS Conference (p. 10). Singapore: World Scientific Publishing.

Oyelude, A. A. (2017, April 3). What's trending in libraries from the internet cybersphere - artificial intelligence and other emerging technologies. Library Hi Tech News.

Sharma, M. (2019). Augmented intelligence: a way for helping universities to make smarter decisions. Emerging Trends in Expert Applications and Security, pp. 89-95.

Upreti, Y. (2021). Issues in Border Security of Nepal. Journal of APF Command and Staff College.

van Teijlingen A, T. T. (2020). Artificial Intelligence and Health in Nepal. Nepal Journal of Epidemiology.

Chan M, Zhen L (2019) Modern military remains a top priority as China boosts defence spending. South China Morning Post

Rayamajhi, P. (2019a, March 18). Nepali Army in nation-building: Quality leadership is the key. The Himalayan Times. .https:// thehimalayantimes.com/opinion/nepali-armyin-nation-buildingquality-leadership-is-thekey/

Stiller, L. F., S. J. (1999). Nepal, Growth of a Nation. (2nd Edition.). Nepal: HRD Research Center 